\title{
An Analysis of Health Status and Long-term Care Expenses of Older Population
}

\author{
Ling-Xue SUN ${ }^{1, a,{ }^{*}}$ \\ ${ }^{1}$ School of Public Affairs, Zhejiang University, \\ Yuhangtang Road 866, 310058 Hangzhou, China \\ asunlx@zju.edu.cn
}

Keywords: Long-term care, Disability dependency ratio, Cost analysis.

\begin{abstract}
This article uses the Zhejiang Province data from the "1\% National Sample Census in $2015 "$ to analyze and predict the health status of the elderly population. Based on the forecast results, this paper defines and measures the disability dependency ratio. As a result, it shows that the disabled population in Zhejiang Province will reach the highest level around 2065, the number is about 4.65 million. The ratio of disability support in 2070 will reach to 0.224 . After that, based on the population forecast, this paper describes the long-term care needs of the disabled and partially disabled people in Zhejiang Province. Finally, the article focuses on the issue of the amount of institutional collection fees in the design of long-term care systems, and measures the fund based on an actuarial perspective. In conclusion, according to the current long-term care system, the corresponding recommendations are given.
\end{abstract}

\section{Introduction}

The number of elderly people in China has been increasing in recent years. According to data from China Statistical Yearbook 2015, in China, the number of people with the age of 65 or older reached 113.75 million in 2014, and the ratio of old-age dependency reached $13.7 \%$. Combined with the data in 2010-2014, we have found that the proportion of the elderly population in the total population is increasing, and the dependency ratio of the elderly is also increasing. How to take good care for the elderly in the future and how to make more effective use of existing resources will become the main topics for the elderly. Among these elderly people, we should focus more on the situation of disabled seniors. Compared to healthy elderly people, disabled seniors often consume more economic and human resources, which also imposes a burden on both their family and the society.

Scholars from various countries have perfected and supplemented the definition of disability. Sidney Katz (1963) first proposed the "ADL Scale," which selected six of the most common operational abilities in daily life as assessment indicators [1]. Florence Mahoney \& Dorothy Barthel (1965) expanded and reformulated the "ADL scale" and later called it the "Barthel Index"[2]. Regarding IADL, Roley et al. (2008) identified 12 occupational care assessment IADL indicators in the article [3]. In addition to the traditional annotations of traditional ADL and IADL judgments, Chen Chuanbo et al. (2013) used the European Five-Dimensional Health Scale (EQ-5D) to determine the degree of disability [4]. The EQ-5D included actions, daily care, daily activities, and pain. And five areas of discomfort, anxiety and depression. Many scholars follow these disability judgment criteria to study disability related issues.

When scholars discussed disability, most articles used the limit dependent variable regression model and analyze as much as possible with longitudinal data. Manton et al. (1988) used longitudinal data to study the changes in the disability status of the elderly in different groups by gender and age [5]. Seeman et al. (2010) used the National Health and Nutrition Examination Surveys (1988-1994 and1999-2004) to find that disability indicators of functional limitations were removed [6]. They observed that other disability conditions increased with age. Payne et al. (2013) used data from Malawi in 2006, 2008, and 2010 to calculate the occurrence of disability transfer [7]. The definition of disability and how to provide long-term care for disabled people is an important 
issue.

\section{Research Method}

\section{Population Health Status Transfer Model}

This study first used the " $1 \%$ National Sample Census in 2015" data from Zhejiang Province to analyze the health status of elderly people in Zhejiang Province by age group in order to predict the amount of long-term care needs. Studies on the factors that influence the disability of the elderly mostly use the Markov method to analyze the status of disability of the elderly. With the age increases, the probability of the elderly getting into disability also increases. However, the elderly can return to one healthy status through care and medical treatment. Among them, the article by Manton, Corder, Stallard (1993) pointed out that ADL disorders can be restored. Therefore, the study used the Sullivan method that keep the proportion of people unchanged [8]. According to the data we use, it classifies the elderly into four grades: health, basic health, unhealthy and disability. The figure 1 shows the difference of health, basic health, unhealthy and disability status from bottom to top with age.

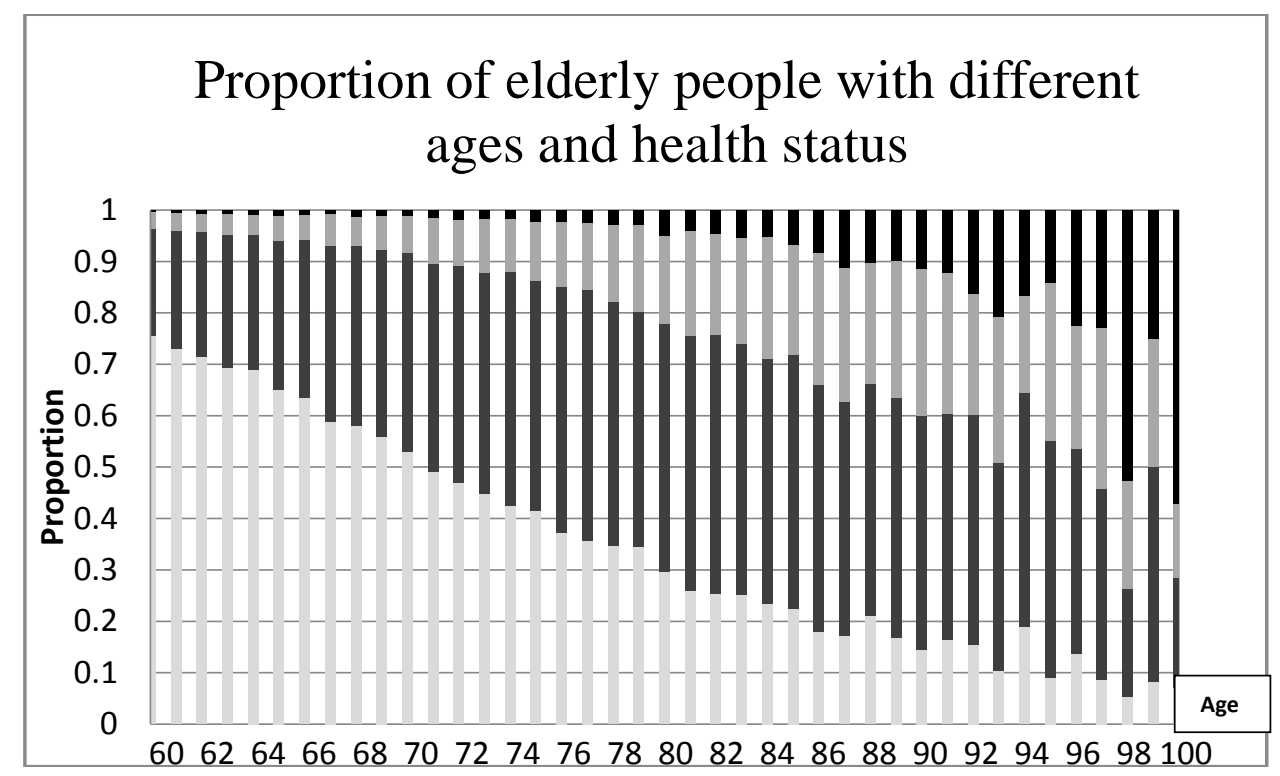

Fig.1 The proportion of elderly people with different ages and health status

\section{Population Forecasting Methods}

In order to study the long-term care system, we use the population forecasting method to obtain the level of disabled people in Zhejiang Province in the future. We find that around the year of 2059, the disabled population in Zhejiang Province will reach the highest level, which is about 4.65 million people. At the same time, for the purpose of combining the long-term care system, we define a new indicator "disability dependency ratio." This indicator refers to the old-age dependency ratio, but it more intuitively reflects the pressure of disabled people given on society. Due to the forecast results, the disability dependency ratio will gradually increase in the future, reaching a maximum of 0.224 in the year of 2070, which means that an average of 4.46 employees will be responsible for one disability old person. At the same time, we also mention that after the year of 2060, the ratio of disability support tends to be stable. The reason is that China's fertility peak gradually withdraw from the historical stage, which brings the population structure slowly changes, and the proportion of the working population has been restored. The figure 2 shows the changes of disability dependency ratio from the year of 2015 to 2069. 


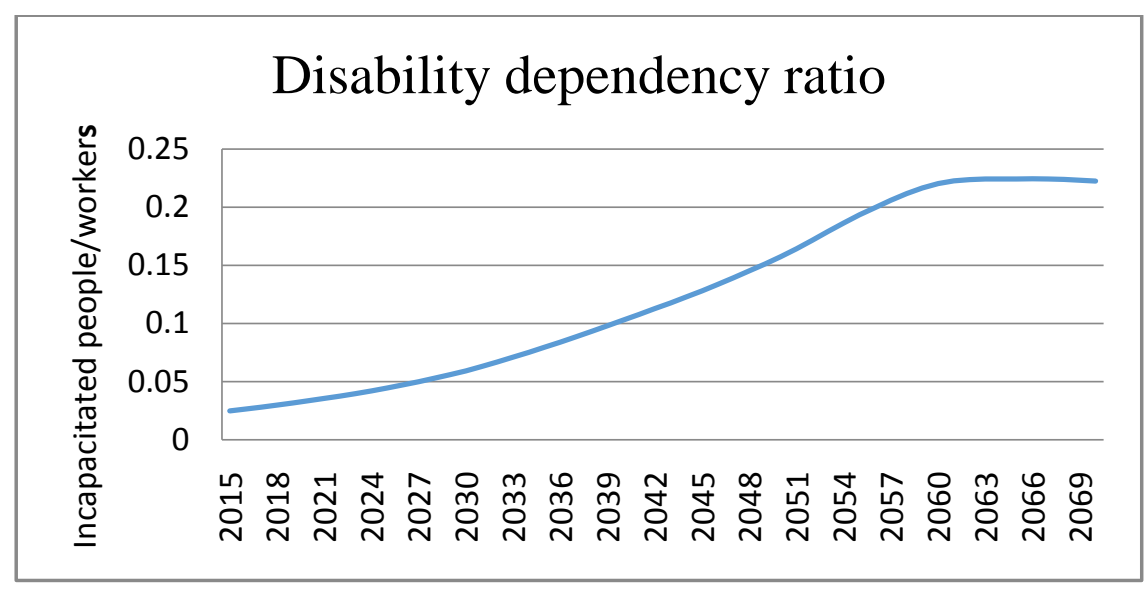

Fig. 2 Disability dependency ratio

\section{Long-Term Care Cost Forecast}

Many scholars have studied the cost of care for disability. When Gu Mengjie (2014) stated the long-term care system of OECD countries [9], he discovered that with the extension of future life expectancy, according to the study of Sven Heinrich and Melanie Luppa (2008), the average costs for male incurred in one year were 3,704 euros and for women were 7,744 euros [10]. In addition to more serious people who must receive formal care, it is also necessary to consider another group of people who choose to informally treat incapacity at home. Their cost is relatively difficult to calculate, but the consumption of the corresponding medical part can be counted. Considering comprehensively, we conservatively estimate that the average long-term care cost per person is 20,000 RMB/year.

The cost of nursing home calculated in their study included the consumption of medical equipment and supplies. Therefore, the consumption index of medical devices and supplies that recorded by the National Bureau of Statistics data was used as the inflation rate for our calculation of future expenditures. In the past 10 years, the geometric mean of the consumption index of medical equipment and supplies in Zhejiang Province is 1.001143332. We use this index as a forecast for the cost of care for future disabled people. We assume that in the future, the annual cost of nursing home will increase according to the above-mentioned proportion, and then we can predict the total annual consumption of all disabled people in 2016-2070 in Zhejiang Province. The figure 3 shows the costs of care for disabled people from the year 2016 to 2070.

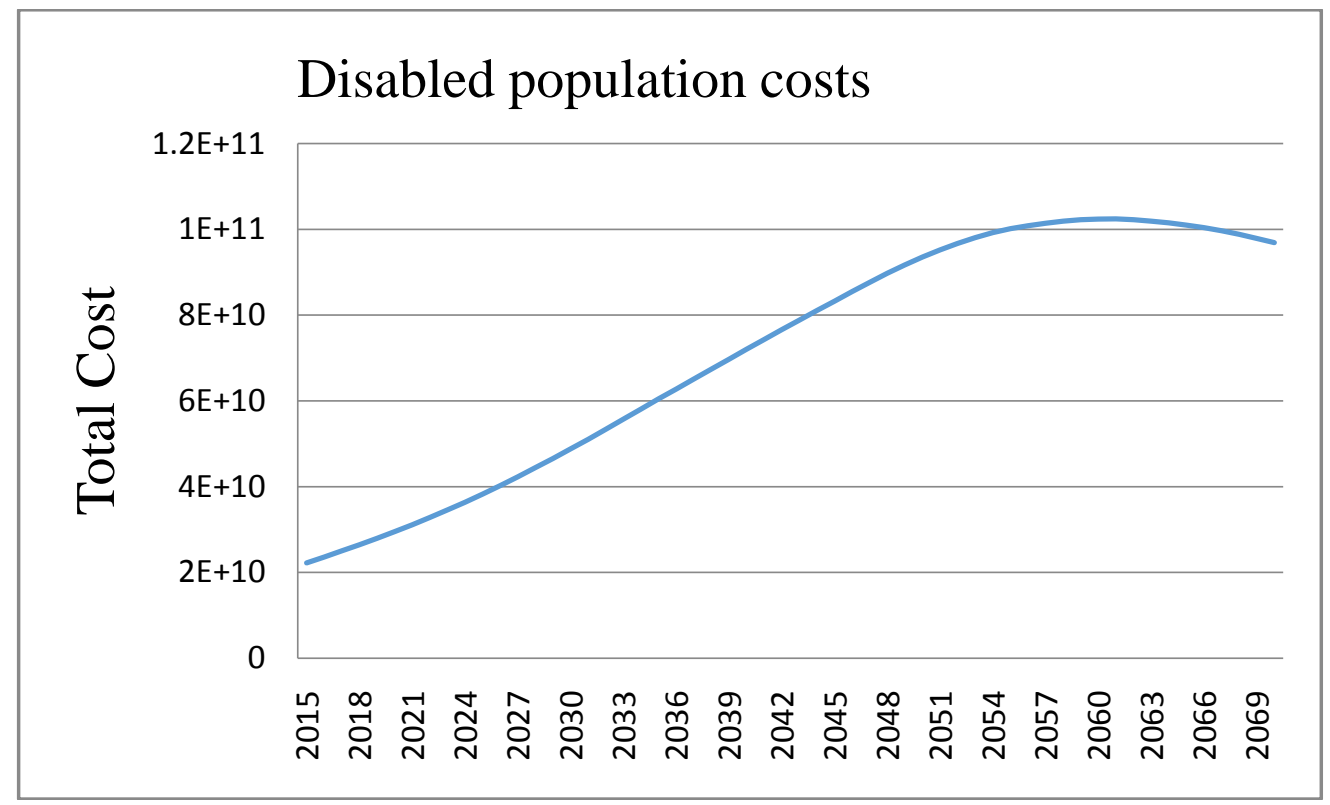

Fig. 3 Costs of care for disabled people 


\section{Multi-State Life Table Application and Payment Model}

As for the long-term care cost forecast, this study proposes a long-term care payment plan design. The following symbols are defined: the interest rate is $r$, the age is $x$, the maximum age is $\omega$; the x-year mortality rate is $q_{x}$; the x-year-old survival number is $l_{x}$, and the x-year-old i state survival number is $l_{x, i}$; The probability of survival t years ${ }_{t} p_{x}$, and the proportion of people in i state $\omega_{i}$, where $\mathrm{i}$ has 2 states, health is a, disability is b, we merge the first two states into a healthy state, the latter two merge is disabled and $\sum_{i} \omega_{i}=1$.

$$
\begin{gathered}
l_{x}=l_{x-1} \cdot\left(1-q_{x-1}\right) \\
l_{x, i}=l_{x} \cdot \omega_{i} \\
{ }_{t} p_{x}=l_{x+t} / l_{x}
\end{gathered}
$$

We assume that the person who is aged $m$ starts to pay for long-term care insurance at the beginning of the year and pays A RMB each year. If these people are incapacitated or die when they pay, we will refund the collected fees. Until the payment of the agreed time $m+k$ years, these people stop paying fees. And from this year onwards, once someone enters a disability state, they will receive an annual insurance of $1 \mathrm{RMB}$ at the beginning of next year. If disabled people recover from disability to health, we will no longer provide insurance money, but will continue to pay once we have returned to disability.

During the period from $\mathrm{m}$ to $\mathrm{m}+\mathrm{k}$, the actuarial present value accumulated by the long-term care insurance funding pool is:

$$
A \cdot \sum_{t=0}^{k} \frac{l_{x+k, a}}{(1+r)^{t}}
$$

Then after $\mathrm{m}+\mathrm{k}+1$ year, the actuarial present value that has been paid to death is:

$$
1 \cdot \sum_{t=k+1}^{\omega} \frac{l_{x+t, b}}{(1+r)^{t}}
$$

According to the principle of actuarial balance, the accumulation of funding pool should be equal to payment.

$$
\begin{gathered}
A \cdot \sum_{t=0}^{k} \frac{l_{x+k, a}}{(1+r)^{t}}=1 \cdot \sum_{t=k+1}^{\omega} \frac{l_{x+t, b}}{(1+r)^{t}} \\
A=\frac{\sum_{t=k+1}^{\omega} \frac{l_{x+k, a}}{(1+r)^{t}}}{\sum_{t=0}^{k} \frac{l_{x+t, b}}{(1+r)^{t}}}
\end{gathered}
$$

Because $\mathrm{k}$ is related to the design of the system, $l_{x}$ can be calculated based on the life table technique. And $\mathrm{r}$ is the interest rate and $\mathrm{P}$ can be calculated either.

Combining with the previous section, we calculated the insurance rate. As a result, each person needs to pay $772.58 \mathrm{RMB}$. Based on this number, we assume a $4 \%$ return on investment. The system will be enforced from the age of 35 in 2016, and they will begin to be guaranteed in 2045 . Income is paid into the crowd each year. In addition to disability payments, the expenses include the refund of the deaths of those who died before the age of 65. Apart from the income and expenditure, the accumulative portion also includes the investment income accumulated in the previous year, and $10 \%$ of them are accumulated reserves cannot be invested.

\section{Summary}

After the year of 2060, with the rise of disabled people in the system and the lack of payment groups, the fund's expenditure will exceed income and begin to decline. It can be predicted that the trend of accumulation of funds in the next 10 years will continue to decline. In 2015, the 35-year-old population will reach 90 years old in 2070. However, in Zhejiang Province, the 
35-year-old male in 2015 has a life expectancy of more than 80 years and females more than 85 . As a result, some of them exceed 90 . The age group does not enter the system coverage and the fund's expenditure will continue to increase. After the fund reaches full coverage, with the restoration of the fertility rate, the working-age population will increase, the ratio of disability support will tend to be stable, and the accumulation of the entire fund will be stable.

We have proposed policy recommendations for establishing a long-term care system for the elderly. While constructing the long-term care system, it is also possible to pay attention to the discussion on delayed retirement in recent years. We find that more than half of the elderly are still in good health before the age of 70, and very few of them are disabled. We can encourage this group of healthy people to postpone retirement. Combined with the long-term care system, we use long-term care cost reductions and other means to encourage these elderly people to serve as care providers in the long-term care system through care giver training. On the one hand it promotes the implementation of delayed retirement policies, and on the other hand it also supplements the human resources of the long-term care system. Considering our country lacks relatively perfect nursing-related laws, the introduction of this system lacks sufficient legal support. This may lead to problems such as non-standard pricing and inconsistent nursing standards. These are all problems that need to be resolved in the future.

\section{References}

[1] Katz, S., Ford, A. B., Moskowitz, R. W., Jackson, B. A., \& Jaffe, M. W. Studies of illness in the aged. The index of adl: A standardized measure of biological and psychosocial function[J]. 1963.Journal of the American Medical Association, 185,914-919.

[2] Florence Mahoney\&Dorothy Barthel. "Functional evaluation: the Barthel Index"[J]. 1965. Md Med J. 14: 61-65.

[3] Roley SS, DeLany JV, Barrows CJ, et al. "Occupational therapy practice framework: domain \& practice, 2nd edition"[J]. 2008.Am J Occup Ther. 62 (6): 625-83.

[4]Chen Chuanbo, Lu Xueqin, Huang Xia, Wei Yanyan, Teng Nan. Investigation and analysis of quality of life and influencing factors of disabled elderly in rural areas[J]. Chinese Journal of Nursing. 2013(08)710-713 (In Chinese)

[5] Kenneth G.Manton, Larry Corder. Eric Stallard, Chronic disability trends in elderly United States populations:1982-1994[J]. 1988.<National Acad Science $>$

[6] Teresa E. Seeman, Sharon S. Merkin, Eileen M. Crimmins, and Arun S. Karlamangla. Disability Trends Among Older Americans: National Health and Nutrition Examination Surveys, 1988-1994 and 1999-2004[J]. <American Journal of Public Health>.2010;100:100-107.

[7]Collin F. Payne, James Mkandawire, Hans-Peter Kohler. Disability Transitions and Health Expectancies among Adults 45 Years and Older in Malawi: A Cohort-Based Model[J]. $<$ PLoS Medicine> May 2013, Volume 10 ,Issue 5

[8] Manton, K.G., Larry Corder. Eric Stallard, 1993, "Estimates of Change in Chronic Disability and Institutional Incidence and Prevalence Rates in the U.S. Elderly Population From the 1982, 1984, and 1989 National Long Term Care Survey.” Journals of Gerontology: Psychological Sciences \& Social Sciences 48: S153-S166.

[9] Gu Mengjie. Research on long-term care allowance system in OECD countries[D]. Anhui Normal University 2014(In Chinese)

[10]Sven Heinrich, Melanie Luppa. Service Utilization and Health-Care Costs in the Advanced Elderly[J].VALUE IN HEALTH,2008,4(11):614. 